\title{
KINETIC DESCRIPTION OF ELECTRON-PROTON INSTABILITY IN HIGH-INTENSITY LINACS AND STORAGE RINGS
}

\author{
Ronald C. Davidson, Hong Qin, and W. Wei-li Lee \\ Plasma Physics Laboratory \\ Princeton University, Princeton, NJ 08543 \\ Tai-Sen F. Wang \\ Los Alamos National Laboratory \\ Los Alamos, NM 87545
}

\begin{abstract}
The Vlasov-Maxwell equations are used to investigate properties of the electron-ion two-stream instability for a continuous, high-intensity ion beam propagating in the $z$ direction with directed axial velocity $V_{b}=\beta_{b} c$ through a background population of (stationary) electrons. The analysis is carried out for arbitrary beam intensity, consistent with transverse confinement of the beam particles, and arbitrary fractional charge neutralization. Stability properties are calculated for dipole perturbations with azimuthal mode number $\ell=1$ about monoenergetic ion and electron distribution functions.
\end{abstract}

\section{INTRODUCTION}

Periodic focusing accelerators and transport systems[1, 2] have a wide range of applications ranging from basic scientific research, to applications such as spallation neutron sources, tritium production, and heavy ion fusion. For a one-component high-intensity beam, considerable progress has been made in describing the self-consistent evolution of the beam distribution function $f_{b}(\mathbf{x}, \mathbf{p}, t)$ and the self-generated electric and magnetic fields in kinetic analyses[1, 3, 4, 5, 6] based on the Vlasov-Maxwell equations. In many practical accelerator applications, however, an (unwanted) second charge component is present. When a second charge component is present, it has been recognized for many years[ $[7,8,9,10,11,12]$ that the relative streaming motion of the high-intensity beam particles through the background charge species provides the free energy to drive the classical two-stream instability[13].

In the present analysis, we apply the Vlasov-Maxwell equations $[1,14]$ to describe the self-consistent interaction of the ion and electron distribution functions with the applied field and the self-generated electric and magnetic fields. The analysis can be applied to ion beams ranging from the emittance-dominated, moderate-intensity proton beams in proton linacs and storage rings, to the lowemittance, space-charge-dominated ion beams in heavy ion fusion.

\section{THEORETICAL MODEL AND ASSUMPTIONS}

The present analysis [14] considers a continuous ion beam with distribution function $f_{b}(\mathbf{x}, \mathbf{p}, t)$, and characteristic radius $r_{b}$ and axial momentum $\gamma_{b} m_{b} \beta_{b} c$ propagating in the $z$-direction through a background population of electrons with distribution function $f_{e}(\mathbf{x}, \mathbf{p}, t)$. The ions have directed axial velocity $V_{b}=\beta_{b} c$, and the background electrons are assumed to be nonrelativistic and stationary with $\int d^{3} p p_{z} f_{e} \simeq 0$ in the laboratory frame. The applied focusing force on a beam ion is modeled by

$$
\mathbf{F}_{f o c}=-\gamma_{b} m_{b} \omega_{\beta b}^{2} \mathbf{x}_{\perp}, \psi
$$

where $\mathbf{x}_{\perp}=x \hat{\mathbf{e}}_{x}+y \hat{\mathbf{e}}_{y}$ is the transverse displacement, $\left(\gamma_{b}-1\right) m_{b} c^{2}$ is the ion kinetic energy, $m_{b}$ is the ion rest mass, $c$ is the speed of light in vacuo, and $\omega_{\beta b}=$ const. is the effective betatron frequency for the applied focusing field. Assuming that the ion density exceeds the background electron density, the space-charge force on an electron, provides transverse confinement of the background electrons by the electrostatic potential $\phi(\mathbf{x}, t)$. It is further assumed that the ion motion in the beam frame is nonrelativistic. The electrostatic potential $\phi(\mathbf{x}, t)$ is determined self-consistently from Poisson's equation $\nabla^{2} \phi=$ $-4 \pi e\left(Z_{b} n_{b}-n_{e}\right)$, and the $z$-component of vector potential $A_{z}(\mathbf{x}, t)$ is determined self-consistently from $\nabla^{2} A_{z}=$ $-4 \pi Z_{b} e \beta_{b} n_{b}$, where the electrons are assumed to carry zero axial current in the laboratory frame. Here, $n_{b}(\mathbf{x}, t)=$ $\int d^{3} p f_{b}(\mathbf{x}, \mathbf{p}, t)$ and $n_{e}(\mathbf{x}, t)=\int d^{3} p f_{e}(\mathbf{x}, \mathbf{p}, t)$ are the ion and electron densities, respectively.

Finally, the stability analysis assumes perturbations with sufficiently long axial wavelength and high frequency that $k_{z}^{2} r_{b}^{2} \ll 1,\left|\omega / k_{z}-\beta_{b} c\right| \gg v_{T b z}$, and $\left|\omega / k_{z}\right| \gg v_{T e z}$. Here, $v_{T b z}=\left(2 T_{b z} / \gamma_{b} m_{b}\right)^{1 / 2}$ and $v_{T e z}=\left(2 T_{e z} / m_{e}\right)^{1 / 2}$ are the axial thermal speeds of the beam ions and the background electrons, respectively. Furthermore, the perturbed axial forces are treated as negligibly small, and the analysis neglects the effects of Landau damping due to axial momentum spread.

We make use of these assumptions to simplify the theoretical model[14]. First, we introduce the reduced distribution functions defined by $F_{j}\left(\mathbf{x}, \mathbf{p}_{\perp}, t\right)=\int d p_{z} f_{j}(\mathbf{x}, \mathbf{p}, t)$ 
for $j=b, e$. Because $\int d p_{z} p_{z} f_{e} \simeq 0$ for the electrons, the nonlinear Vlasov equation for $F_{e}\left(\mathbf{x}, \mathbf{p}_{\perp}, t\right)$ is given by

$$
\left\{\frac{\partial}{\partial t}+\frac{\mathbf{p}_{\perp}}{m_{e}} \cdot \frac{\partial}{\partial \mathbf{x}_{\perp}}+\left(e \nabla_{\perp} \phi\right) \cdot \frac{\partial}{\partial \mathbf{p}_{\perp}}\right\} F_{e}\left(\mathbf{x}, \mathbf{p}_{\perp}, t\right)=0
$$

where $-e$ is the electron charge, and $\nabla_{\perp} \equiv \hat{\mathbf{e}}_{x} \partial / \partial x+$ $\hat{\mathbf{e}}_{y} \partial / \partial y$. The ions, however, have large directed axial velocity $V_{b} \simeq \beta_{b} c$, and the Vlasov equation for $F_{b}\left(\mathbf{x}, \mathbf{p}_{\perp}, t\right)$ becomes

$$
\begin{aligned}
& \left\{\frac{\partial}{\partial t}+V_{b} \frac{\partial}{\partial z}+\frac{\mathbf{p}_{\perp}}{\gamma_{b} m_{b}} \cdot \frac{\partial}{\partial \mathbf{x}_{\perp}}-\left(\gamma_{b} m_{b} \omega_{\beta b}{ }^{2} \mathbf{x}_{\perp}\right.\right. \\
& \left.\left.+Z_{b} e \nabla_{\perp} \psi\right) \cdot \frac{\partial}{\partial \mathbf{p}_{\perp}}\right\} F_{b}\left(\mathbf{x}, \mathbf{p}_{\perp}, t\right)=0 .
\end{aligned}
$$

Here, $+Z_{b} e$ is the ion charge, and $\psi(\mathbf{x}, t) \equiv \phi(\mathbf{x}, t)-$ $\beta_{b} A_{z}(\mathbf{x}, t)$. The self-field potentials $\phi(\mathbf{x}, t)$, and $\psi(\mathbf{x}, t)$ in Eqs. (2) and (3) are determined self-consistently from

$$
\begin{aligned}
& \left(\frac{\partial^{2}}{\partial x^{2}}+\frac{\partial^{2}}{\partial y^{2}}\right) \phi=-4 \pi e\left(Z_{b} \int d^{2} p F_{b}-\int d^{2} p F_{e}\right), \\
& \left(\frac{\partial^{2}}{\partial x^{2}}+\frac{\partial^{2}}{\partial y^{2}}\right) \psi=-4 \pi e\left(\frac{Z_{b}}{\gamma_{b}^{2}} \int d^{2} p F_{b}-\int d^{2} p F_{e}\right) .
\end{aligned}
$$

We assume that the beam propagates axially through a perfectly conducting cylindrical pipe with radius $r=r_{w}$. Enforcing $\left[E_{\theta}^{s}\right]_{r=r_{w}}=\left[E_{z}^{s}\right]_{r=r_{w}}=\left[B_{r}^{s}\right]_{r=r_{w}}=0$ readily gives $\phi\left(r=r_{w}, \theta, z, t\right)=0$, and $\psi\left(r=r_{w}, \theta, z, t\right)=0$. Here, the constant values of the potentials at $r=r_{w}$ have been taken equal to zero.

\section{EQUILIBRIUM PROPERTIES}

Under quasisteady equilibrium conditions with $\partial / \partial t=0$, we assume axisymmetric beam propagation and negligible variation with axial coordinate $(\partial / \partial \theta=0=\partial / \partial z)$. The equilibrium distribution functions for the beam ions and background electrons are of the general form $F_{b}^{0}=$ $F_{b}^{0}\left(H_{\perp b}\right)$ and $F_{e}^{0}=F_{e}^{0}\left(H_{\perp e}\right)$, where

$$
\begin{aligned}
& H_{\perp b}=\frac{1}{2 \gamma_{b} m_{b}} \mathbf{p}_{\perp}^{2}+\frac{1}{2} \gamma_{b} m_{b} \omega_{\beta b}^{2} r^{2}+Z_{b} e\left[\psi^{0}(r)-\hat{\psi}^{0}\right], \\
& H_{\perp e}=\frac{1}{2 m_{e}} \mathbf{p}_{\perp}^{2}-e\left[\phi^{0}(r)-\hat{\phi}^{0}\right] .
\end{aligned}
$$

Here, $r=\left(x^{2}+y^{2}\right)^{1 / 2}$ is the radial distance from the beam axis, $H_{\perp b}$ and $H_{\perp e}$ are exact single-particle constants of the motion, and $\hat{\psi}^{0} \equiv \psi^{0}(r=0)$ and $\hat{\phi}^{0} \equiv \phi^{0}(r=0)$ are constants.

There is wide latitude in specifying the functional forms of the equilibrium distribution functions[14]. In the present analysis, we assume monoenergetic ions and electrons, with distribution functions

$$
\begin{aligned}
& F_{b}^{0}\left(H_{\perp b}\right)=\frac{\hat{n}_{b}}{2 \pi \gamma_{b} m_{b}} \delta\left(H_{\perp b}-\hat{T}_{\perp b}\right), \\
& F_{e}^{0}\left(H_{\perp e}\right)=\frac{\hat{n}_{e}}{2 \pi m_{e}} \delta\left(H_{\perp e}-\hat{T}_{\perp e}\right) .
\end{aligned}
$$

Here, $\hat{n}_{b}$ and $\hat{n}_{e} \equiv f Z_{b} \hat{n}_{b}$ are positive constants corresponding to the ion and electron densities, $f=$ const. is the fractional charge neutralization, and $\hat{T}_{\perp b}$ and $\hat{T}_{\perp e}$ are constants corresponding to the on-axis $(r=0)$ values of the transverse ion and electron temperatures, respectively. Without presenting details[14], some algebraic manipulation of Eqs. (4) - (6) gives the step-function density profiles $n_{j}^{0}(r)=\hat{n}_{j}=$ const., for $0 \leq r<r_{b}$, and $n_{j}^{0}(r)=0$ for $r_{b}<r \leq r_{w}$, and $j=b, e$. Here, the beam radius $r_{b}$ is related to other equilibrium parameters by $\hat{\nu}_{b}^{2} r_{b}^{2}=2 \hat{T}_{\perp b} / \gamma_{b} m_{b}$ and $\hat{\nu}_{e}^{2} r_{b}^{2}=2 \hat{T}_{\perp e} / m_{e}$, where the (depressed) betatron frequencies $\hat{\nu}_{b}$ and $\hat{\nu}_{e}$ are defined by

$$
\begin{aligned}
& \hat{\nu}_{b}^{2}=\omega_{\beta b}^{2}-\frac{1}{2}\left(\frac{1}{\gamma_{b}^{2}}-f\right) \hat{\omega}_{p b}^{2}=\text { const. } \\
& \hat{\nu}_{e}^{2}=\frac{1}{2} \frac{\gamma_{b} m_{b}}{Z_{b} m_{e}}(1-f) \hat{\omega}_{p b}^{2}=\text { const. }
\end{aligned}
$$

and $\hat{\omega}_{p b}^{2}=4 \pi \hat{n}_{b} Z_{b}^{2} e^{2} / \gamma_{b} m_{b}$ is the ion plasma frequencysquared.

\section{STABILITY ANALYSIS AND DISPERSION RELATION}

For small-amplitude perturbations, a stability analysis proceeds by linearizing Eqs. (2)-(4). Perturbed quantities are expressed as $\delta \psi(\mathbf{x}, t)=\delta \hat{\psi}^{l}(r) \exp \left(i k_{z} z+i l \theta-i \omega t\right)$, $\delta F_{b}\left(\mathbf{x}, \mathbf{p}_{\perp}, t\right)=\delta \hat{F}_{b}^{l}\left(r, \mathbf{p}_{\perp}\right) \exp \left(i k_{z} z+i l \theta-i \omega t\right)$, etc. where $I m \omega>0$ is assumed, corresponding to instability (temporal growth), $k_{z}$ is the axial wavenumber, and $l$ is the azimuthal harmonic number. The linearized Vlasov equations are formally integrated by using the method of characteristics[14]. For perturbations about the monoenergetic ion and electron distribution functions in Eq. (6), we obtain[14] the kinetic dispersion relation

$$
\begin{aligned}
& {\left[\frac{2}{1-\left(r_{b} / r_{w}\right)^{2 \ell}}+\frac{\hat{\omega}_{p b}^{2}}{\ell \gamma_{b}^{2} \hat{\nu}_{b}^{2}} \Gamma_{b}^{\ell}\left(\omega-k_{z} V_{b}\right)\right]\left[\frac{2}{1-\left(r_{b} / r_{w}\right)^{2 \ell}}\right.} \\
& \left.+\frac{\hat{\omega}_{p e}^{2}}{\ell \hat{\nu}_{e}^{2}} \Gamma_{e}^{\ell}(\omega)\right]=\frac{\hat{\omega}_{p e}^{2}}{\ell \hat{\nu}_{e}^{2}} \cdot \frac{\hat{\omega}_{p b}^{2}}{\ell \hat{\nu}_{b}^{2}} \Gamma_{e}^{\ell}(\omega) \Gamma_{b}^{\ell}\left(\omega-k_{z} V_{b}\right) .
\end{aligned}
$$

where $\hat{\omega}_{p e}^{2}=4 \pi \hat{n}_{e} e^{2} / m_{e}$. Here, the ion and electron susceptibilities are defined by[14]

$$
\begin{aligned}
& \Gamma_{b}^{\ell}\left(\omega-k_{z} V_{b}\right)=-\frac{1}{2^{\ell}} \sum_{m=0}^{\ell} \frac{\ell !}{m !(\ell-m) !} \times \\
& \frac{(\ell-2 m) \hat{\nu}_{b}}{\left[\left(\omega-k_{z} V_{b}\right)-(\ell-2 m) \hat{\nu}_{b}\right]}, \\
& \Gamma_{e}^{\ell}(\omega)=-\frac{1}{2^{\ell}} \sum_{m=0}^{\ell} \frac{\ell !}{m !(\ell-m) !} \frac{(\ell-2 m) \hat{\nu}_{e}}{\left[\omega-(\ell-2 m) \hat{\nu}_{e}\right]}
\end{aligned}
$$

for general azimuthal harmonic number $l$.

A careful examination[14] of Eq. (8) for $\hat{n}_{e} \neq 0$ shows that the strongest instability (largest growth rate) occurs for azimuthal mode number $\ell=1$, corresponding to a simple 
(dipole) displacement of the beam ions and the background electrons. For $\ell=1$, we find $\Gamma_{e}^{1}(\omega)=-\hat{\nu}_{e}^{2} /\left[\omega^{2}-\hat{\nu}_{e}^{2}\right]$ and $\Gamma_{b}^{1}\left(\omega-k_{z} V_{b}\right)=-\hat{\nu}_{b}^{2} /\left[\left(\omega-k_{z} V_{b}\right)^{2}-\hat{\nu}_{b}^{2}\right]$, and introduce the collective oscillation frequencies defined by

$\omega_{e}^{2} \equiv \hat{\nu}_{e}^{2}+\frac{1}{2} \hat{\omega}_{p e}^{2}\left(1-\frac{r_{b}^{2}}{r_{w}^{2}}\right)=\frac{1}{2} \frac{\gamma_{b} m_{b}}{Z_{b} m_{e}} \hat{\omega}_{p b}^{2}\left(1-f \frac{r_{b}^{2}}{r_{w}^{2}}\right)$

$\omega_{b}^{2} \equiv \hat{\nu}_{b}^{2}+\frac{\hat{\omega}_{p b}^{2}}{2 \gamma_{b}^{2}}\left(1-\frac{r_{b}^{2}}{r_{w}^{2}}\right)=\omega_{\beta b}^{2}+\frac{1}{2} \hat{\omega}_{p b}^{2}\left(f-\frac{1}{\gamma_{b}^{2}} \frac{r_{b}^{2}}{r_{w}^{2}}\right)$

where use is made of $\hat{\omega}_{p e}^{2}=\left(\gamma_{b} m_{b} / Z_{b} m_{e}\right) f \hat{\omega}_{p b}^{2}$. Substituting into Eq. (8) and rearranging terms, the $\ell=1$ dispersion relation can be expressed in the compact form

$$
\left[\left(\omega-k_{z} V_{b}\right)^{2}-\omega_{b}^{2}\right]\left[\omega^{2}-\omega_{e}^{2}\right]=\omega_{f}^{4},
$$

where $\omega_{f}$ is defined by

$$
\omega_{f}^{4} \equiv \frac{1}{4} f\left(1-\frac{r_{b}^{2}}{r_{w}^{2}}\right)^{2} \frac{\gamma_{b} m_{b}}{Z_{b} m_{e}} \hat{\omega}_{p b}^{4} .
$$

In the absence of background electrons ( $f=0$ and $\omega_{f}=0$ ), Eq. (11) gives stable collective oscillations of the ion beam with frequency $\omega-k_{z} V_{b}= \pm \omega_{b}$, where $\omega_{b}$ is defined in Eq. (11). For $f \neq 0$, however, the ion and electron terms on the left-hand side of Eq. (11) are coupled by the $\omega_{f}^{4}$ term on the right-hand side, leading to one unstable solution with $\operatorname{Im} \omega>0$ for a certain range of axial wavenumber $k_{z}$. It is important to recognize that the dispersion relation (11) is applicable over a wide range of normalized beam intensity and fractional charge neutralization. That is, Eq. (11) can be applied to the emittancedominated, moderate-intensity ion beams $\left(\hat{\omega}_{p b}^{2} / \omega_{\beta b}^{2} \lesssim 0.2\right.$, say) in proton linacs and storage rings. On the other hand, Eq. (11) can also be applied to the low-emittance, very high-intensity ion beams $\left(\hat{\omega}_{p b}^{2} / \omega_{\beta b}^{2}\right.$ approaching $2 \gamma_{b}^{2}$, for $f=0$ ) envisioned for heavy ion fusion.

A careful examination of Eq. (11) shows that the unstable, positive-frequency branch has frequency and wavenumber $\left(\omega, k_{z}\right)$ closely tuned to the values $\left(\omega_{0}, k_{z 0}\right)$ defined by $\omega_{0}=+\omega_{e}$ and $\omega_{0}-k_{z 0} V_{b}=-\omega_{b}$. In this regime, expressing $\omega=\omega_{0}+\delta \omega$ and $k_{z}=k_{z 0}+\delta k_{z}$, and assuming $|\delta \omega| \ll 2 \omega_{e}$, the dispersion relation (11) is given to good approximation by

$$
\begin{aligned}
& \delta \omega\left(\delta \omega-\delta k_{z} V_{b}\right)\left[1-\left(\delta \omega-\delta k_{z} V_{b}\right) / 2 \omega_{b}\right] \\
& =-\Gamma_{0}^{2} \equiv-\frac{\omega_{f}^{4}}{4 \omega_{e} \omega_{b}} .
\end{aligned}
$$

At moderate beam intensities with $\Gamma_{0}^{2} \ll 1$, the unstable solution to Eq. (13) satisfies $\left|\delta \omega-\delta k_{z} V_{b}\right| \ll 2 \omega_{b}$. In this regime, Eq. (13) can be approximated by the quadratic form $\delta \omega\left(\delta \omega-\delta k_{z} V_{b}\right)=-\Gamma_{0}^{2} \equiv-\omega_{f}^{4} / 4 \omega_{e} \omega_{b}$. This quadratic dispersion relation supports an unstable solution with growth rate $\operatorname{Im} \delta \omega=\Gamma_{0}\left[1-\left(\delta k_{z} V_{b} / 2 \Gamma_{0}\right)^{2}\right]^{1 / 2}$ for $\delta k_{z}$ in the (symmetric) interval, $-2 \Gamma_{0}<\delta k_{z} V_{b}<$ $2 \Gamma_{0}$. The maximum growth rate is $(\operatorname{Im} \delta \omega)_{\max }=\Gamma_{0} \equiv$ $\omega_{f}^{2} / 2\left(\omega_{e} \omega_{b}\right)^{1 / 2}$, which occurs for $\delta k_{z}=0$. For example, for a proton beam $\left(Z_{b}=1, m_{b} / m_{e}=1836\right)$ with relativistic mass factor $\gamma_{b}=1.85$, a moderate value of normalized beam intensity $\hat{\omega}_{p b}^{2} / \omega_{\beta b}^{2}=0.1$, large wall radius $r_{w} / r_{b} \rightarrow \infty$ and fractional charge neutralization $f=0.1$, we obtain $(\operatorname{Im} \delta \omega)_{\max }=0.127 \omega_{\beta b}$, corresponding to a particularly virulent growth rate for the electronproton (e-p) instability. For this choice of system parameters, the central oscillation frequency and wavenumber are $\omega_{0}=13.03 \omega_{\beta b}$ and $k_{z 0} V_{b}=14.03 \omega_{\beta b}$.

\section{CONCLUSIONS}

The general kinetic formalism[14] outlined here can also be applied to perturbations about a wide range of nonmonoenergetic equilibrium distribution functions. A detailed, self-consistent stability analysis based on Eqs. (2)(4) for continuously varying profiles is beyond the scope of the present article. It is sufficient to note that the spread in (depressed) betatron frequencies[7, 14] associated with continuously varying profiles is expected to lead to a threshold in beam intensity and/or fractional charge neutralization for the onset of instability.

\section{ACKNOWLEDGEMENT}

This research was supported by the Department of Energy and the APT Project and LANSCE Division of Los Alamos National Laboratory.

\section{REFERENCES}

[1] R. C. Davidson, Physics of Nonneutral Plasmas (AddisonWesley Publishing Co., Reading, MA, 1990), and references therein.

[2] T. P. Wangler, Principles of RF Linear Accelerators (John Wiley \& Sons, Inc., New York, 1998).

[3] T. -S. Wang and L. Smith, Part. Accel. 12, 247 (1982).

[4] J. Struckmeier and I. Hofmann, Part. Accel. 39, 219 (1992).

[5] R. C. Davidson, Physics of Plasmas 5, 3459 (1998).

[6] R. C. Davidson and C. Chen, Part. Accel. 59, 175 (1998).

[7] D. Koshkarev and P. Zenkevich, Part. Accel. 3, 1 (1972).

[8] E. Keil and B. Zotter, CERN-ISR-TH/71-58 (1971).

[9] L. J. Laslett, A. M. Sessler, and D. Möhl, Nuclear Instruments and Methods 121, 517 (1974).

[10] D. Neuffer, E. Colton, D. Fitzgerald, T. Hardek, R. Hutson, R. Macek, M. Plum, H. Thiessen, and T. -S. Wang, Nuclear Instruments and Methods in Phys. Res. A321, 1 (1992).

[11] J. Byrd, A. Chao, S. Heifets, M. Minty, T. O. Roubenheimer, J. Seeman, G. Stupakov, J. Thomson and F. Zimmerman, Phys. Rev. Lett. 79, 79 (1997).

[12] K. Ohmi, Phys. Rev. E55, 7550 (1997).

[13] See, for example, pp. 240-271 of Ref. 1.

[14] "Kinetic Description of Electron-Proton Instability in HighIntensity Proton Linacs and Storage Rings Based on the Vlasov-Maxwell Equations," R. C. Davidson, H. Qin, P. H. Stoltz, and T. -S. Wang, submitted for publication (1999). 